\title{
Eribulin: Neue Therapieoption für intensiv vorbehandelte Patientinnen
}

\begin{abstract}
Seit Mai 2011 steht mit Eribulin ein neues Zytostatikum für die Behandlung von Patientinnen mit Mammakarzinom zur Verfügung, das in Studien auch bei Patientinnen mit lokal fortgeschrittenem oder metastasiertem Mammakarzinom und intensiver Vorbehandlung zur Überlebensverlängerung führte.
\end{abstract}

Eribulin, ein synthetisch hergestelltes Analogon des aus dem pazifischen Meeresschwamm Halichondria okadai gewonnenen Halichondrin B, hemmt ähnlich wie die Taxane und Vincaalkaloide die Zellteilung, erläuterte PD Dr. Sibylle Loibl, Neu-Isenburg. Der neuartige Wirkungsmechanismus - Eribulin (Halaven ${ }^{\oplus}$ ) behindert den Aufbau der Mikrotubuli, verkürzt sie dabei aber nicht - wird als Ursache dafür diskutiert, dass es auch bei Tumoren wirkt, die auf eine Taxanbehandlung nicht mehr ansprechen.

Eribulin hat von der Europäischen Kommission die Zulassung für die Behandlung von Patientinnen mit lokal fortgeschrittenem oder metastasiertem Mammakarzinom erhalten, deren Zustand sich nach mindestens zwei Behandlungszyklen Chemotherapie im fortgeschrittenen Stadium weiter verschlechtert hat. Die Vortherapien sollten dabei - sofern für die Patientinnen geeignet - ein Anthrazyklin und ein Taxan enthalten haben.
Basis der EU-weiten Zulassung: Phase-III-Studie EMBRACE In die Phase-III-Studie EMBRACE (Eisai Metastatic Breast Cancer Study Assessing Treatment of Physician's Choice [TPC] Versus Eribulin E7389) waren 762 Patientinnen mit lokal fortgeschrittenem oder metastasiertem Mammakarzinom im Alter von 27 bis 85 Jahren eingeschlossen, die bereits vier Chemotherapien - darunter ein Anthrazyklin und ein Taxan - erhalten hatten (Cortes J et al., 2011, Lancet 377: 914-923). Die Patientinnen wurden im Verhältnis 2:1 randomisiert mit Eribulin als Monotherapie $\left(1,23 \mathrm{mg} / \mathrm{m}^{2}\right.$ KOF) oder einer konventionellen Chemotherapie nach Wahl des Arztes behandelt.

Durch den Einsatz von Eribulin konnte das mediane Gesamtüberleben signifikant von 10,6 Monaten auf 13,1 Monate verlängert werden (Hazard-Ratio [HR] 0,81; 95-\%-Konfidenzintervall $0,66-0,99 ; p=0,041)$. „Eine von der europäischen und amerikanischen Aufsichtsbehörde geforderte aktualisierte Analyse nach 589 Todesfällen bestätigte den positiven Effekt auf das Gesamtüberleben", sagte Loibl (medianes Gesamtüberleben: 13,2 Monate versus 10,5 Monate; HR 0,81; nominal $p=0,014$; Twelves $C$ et al., 2011, Cancer Res 70 [Suppl]: P6-14-08). AY

Quelle: Veranstaltung der Eisai $\mathrm{GmbH}$

\section{Herpes zoster}

\section{Rechtzeitiger Therapiebeginn ist entscheidend}

Das Ziel der Zostertherapie ist es, die akute Krankheitsphase zu verkürzen und Komplikationen vorzubeugen.

Für immunkompetente Erwachsene ist die orale systemische antivirale Therapie mit Aciclovir, Brivudin, Famciclovir oder Valaciclovir indiziert (RKI-Ratgeber Infektionskrankheiten, Merkblätter für Ärzte, 2010, Varizellen, Herpes zoster). Aciclovir parenteral sollte bei Immunsupprimierten und bei Komplikationen, wie Zoster ophthalmicus, verabreicht werden. Die orale Gabe von Famciclovir ist ebenso möglich.

Die Virustatika sind in ihrer Wirksamkeit auf kutane Zosterläsionen nahezu gleichwertig (Wutzler P et al., 2003, Dtsch Arztebl, 100: A858-A861). Die Dauer des zosterassoziierten Schmerzes ist bei Brivudin, Famciclovir und Valaciclovir verglichen mit Aciclovir vermindert. Einen Vorteil hat Brivudin durch die Einmaldosierung pro Tag für sieben Tage verglichen mit Aciclovir, Famciclovir oder Valaciclovir (Fachinformation Zostex ${ }^{\circledR}$, Stand Oktober 2010). Brivudin darf nicht zur Behandlung eingesetzt werden, wenn die Immunabwehr stark eingeschränkt ist, z.B. durch eine zytostatische Chemotherapie, durch eine immunsuppressive Therapie oder durch die Behandlung einer schweren generalisierten Pilzerkrankung mit Flucytosin. Brivudin und 5-Fluorouracil (5-FU), einschließlich topisch anzuwendender 5-FU-Zubereitungen, oder 5-FU-Prodrugs (z.B. Capecitabin, Floxuridin, Tegafur) oder Kombinationsarzneimittel mit diesen Wirkstoffen oder andere 5-Fluoropyrimidine (z.B. Flucytosin) dürfen nicht zusammen verabreicht werden. Weiterhin muss zwischen einer Behandlung mit Brivudin und dem Beginn einer Therapie mit 5-Fluoropyrimidin-haltigen Arzneimitteln ein zeitlicher Abstand von mindestens vier Wochen eingehalten werden.

Quelle: Informationen der Berlin-Chemie AG
Therapie Erlotinibinduzierter Hautreaktionen: Dermatologische Referenzzentren online

Zwei Drittel der Patienten, die aufgrund eines fortgeschrittenen Pankreas- oder Lungenkarzinoms mit dem EGFR-Inhibitor Erlotinib (Tarceva ${ }^{\circledast}$, Roche Pharma AG) behandelt werden, entwickeln ein papulopustulöses Exanthem (Rash). Die Stärke der Hautreaktion korreliert dabei positiv mit dem Therapieansprechen. Sehr starker Rash kann für den Patienten jedoch eine erhebliche Belastung darstellen. Nicht selten wird dann die Dosis reduziert oder die Therapie abgebrochen. Um den Therapieerfolg mit Erlotinib in diesen Fällen nicht zu gefährden, empfiehlt sich die Überweisung des Patienten an ein dermatologisches Referenzzentrum. Eine Liste aller Zentren kann ab sofort unter www. tarceva.de im passwortgeschützten Bereich abgerufen werden. 